\title{
Algorithm for P versus NP Problem on Sets by JEEVAN - KUSHALAIAH Method
}

\author{
Neelam Jeevan Kumar \\ Electrical and Electronics Engineering, JNTU Hyderabad \\ Rangashaipet, Warangal, Andhra Pradesh, India.
}

\begin{abstract}
P versus NP [1-2] Problems are one of the most important open questions in mathematics and theoretical computer science. Jeevan - Kushalaiah Method is a method to find the possible number of combinations between n-elements. This article explains about Algorithm to solve subset sum problem quickly and easily. The problems on subset sum problems perform arithmetic operations that can be calculated in terms of exponential time - polynomial time. Major part of the article deals with Class-P type problems which in be solved on a deterministic Turing Machine. This article is mainly prepared on the basis of an article in Wikipedia.
\end{abstract}

Keywords: Jeevan-Kushalaiah Method; Jeevan - Kushalaiah Algorithm; Time Complexity; Exponential Time; Polynomial Time;

\section{INTRODUCTION}

In computational complexity theory an answer to the $\mathrm{P}=\mathrm{NP}$ [3-4] question would determine whether problems that can be verified in polynomial time, like the subset-sum problem, can also be solved in exponential- polynomial time. If it turned out that $\mathrm{P} \neq$ $\mathrm{NP}$, it would mean that there are problems in NP (such as NP-complete problems) that are harder to compute than to verify: they could not be solved in polynomial time, but the answer could be verified in polynomial time. The algorithm named as Jeevan - Kushalaiah Algorithm because it uses Jeevan-Kushalaiah Method. The Time taken to produce the output is in Exponential -polynomial time

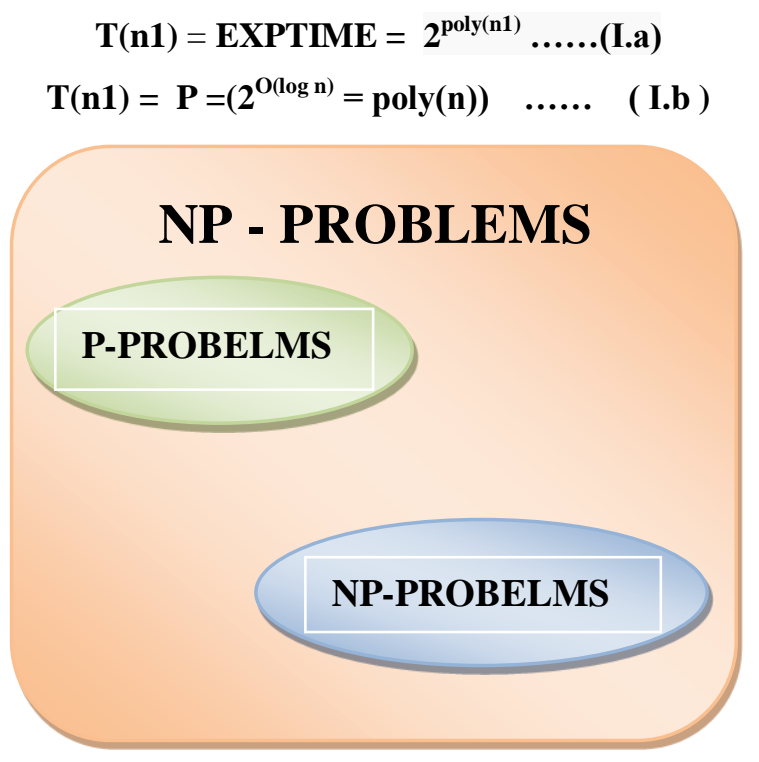

Figure.1: Diagram of complexity classes provided that $\mathrm{P} \neq \mathrm{NP}$. The existence of problems within NP but outside both $\mathrm{P}$ and NP-complete, under that assumption, was established by Ladner's theorem

\section{JEEVAN - KUSHALAIAH METHOD}

Jeevan - Kushalaiah Method [5] is a method to know possible different combinations between nelements (either elements are constants or variables).

Let there are n-elements $\mathbf{a}_{1}, \mathbf{a}_{2}, \mathbf{a}_{3} \ldots \mathbf{a}_{\mathbf{n}}$

The possible number of Combinations are in sets with addition operation are, $\mathrm{N}$

$$
\begin{aligned}
& N=\left\{0,\left[\left(a_{1}\right),\left(a_{2}\right),\left(a_{3}\right), \ldots .\left(a_{2}\right)\right],\left[\left(a_{1}+a_{2}\right),\left(a_{1}+a_{3}\right),\left(a_{1}+\right.\right.\right. \\
& \left.a_{4}\right), \ldots \ldots\left(a_{1}+a_{n}\right),\left(a_{2}+a_{3}\right),\left(a_{2}+a_{4}\right),\left(a_{2}+a_{5}\right), \ldots \\
& \left(a_{2}+a_{n}\right),\left(a_{3}+a_{4}\right), \quad \ldots\left(a_{3}+a_{n}\right), \ldots .\left(a_{n-}\right. \\
& \left.\left.{ }_{1}+a_{n}\right)\right],\left[\left(a_{1}+a_{2}+a_{3}\right),\left(a_{1}+a_{2}+a_{4}\right),\left(a_{1}+a_{2}+a_{5}\right),\right. \\
& \left.\left.+a_{n-1}+a_{n}\right)\right] \ldots \ldots . . \ldots\left(a_{n-2}\right.
\end{aligned}
$$

$$
\begin{aligned}
& \mathrm{N}=\left\{\Theta_{0}, \Theta_{1}, \Theta_{2}, \Theta_{\mathrm{n}-1}, \Theta_{\mathrm{n}}\right\} \\
& \text { Where } \Theta_{1}=\left[\left(a_{1}\right),\left(a_{2}\right),\left(a_{3}\right), \ldots .\left(a_{2}\right)\right] \quad \ldots . . . \quad \Theta_{n} \\
& =\left[\left(a_{1}+a_{2}+a_{3}+\ldots+a_{n}\right)\right] \\
& \Theta_{\mathrm{n}}=\left[\phi_{\mathrm{n}, 1}, \phi_{\mathrm{n}, 2}, \ldots \Phi_{\mathrm{n}, \mathrm{p}}\right]
\end{aligned}
$$$$
\text { Maximum value of } \phi_{\mathrm{n}, \mathrm{p}} \text { is }
$$

$$
\begin{gathered}
\Phi_{n, p}={ }^{n} C_{p}=\left(\begin{array}{l}
n \\
p
\end{array}\right)=(n !) /(n-p) !(p) ! \\
\boldsymbol{\Theta}_{n=} \sum_{i=1}^{\left(\begin{array}{l}
n \\
p
\end{array}\right)} \Phi_{n},\left(\begin{array}{l}
n \\
p
\end{array}\right) \ldots \ldots .
\end{gathered}
$$

The possible number of Combinations, $\mathrm{N}$

$$
\mathbf{N}=\sum_{\mathrm{i}=\mathbf{1}}^{\mathrm{n}} \boldsymbol{\theta}_{\mathrm{n}} \ldots \ldots(\mathrm{IV})
$$




\section{JEEVAN - KUSHALAIAH ALGORITHM}

To find Subset of Set is Predefined value, $\mathrm{x}$ by adding elements of Set. To find which subset combination has given predefined value by adding has to Jeevan-Kushalaiah Algorithm.

Jeevan - Kushalaiah Algorithm must satisfy three conditions on Sets before going to algorithm process.

Condition-1: Create Subsets with positive and negative numbers and name as $P_{n}$ and $N_{n}$ respectively

Condition-2: The sum all values of either set should not be less than minimum value of other set

$\operatorname{Mod}\left\{\sum \mathbf{P}_{\mathbf{n}}\right\} \operatorname{not}<\operatorname{Mod}\left\{\min \left[\mathbf{N}_{\mathbf{n}}\right]\right\} \quad$ or $\operatorname{Mod}\left\{\sum \mathbf{N}_{\mathbf{n}}\right\} \operatorname{not}<\operatorname{Mod}\left\{\min \left[\mathbf{P}_{\mathbf{n}}\right]\right\}$

Condition-3: Eliminate or remove or delete $\Theta_{0}$ value from $P_{n}$ and $N_{n}$

Condition-4: The set should not contain all same sign numbers

The algorithm steps:
Step-1: Start the Program

Step-2: Perform condition-1 on given set.

Step-3: Create possible number of subsets in $P_{n}, N_{n}$ with equations (II),(III) and (IV)

Step-4:Check condition-2 if it fails display output as 'NOT POSSIBLE' then go to step-10 otherwise go to step-5

Step-5: Set $Z=0$, Where $Z$ is the number of Predefined valued subsets.

Step-6: Add each subset of $P_{n}\left(\right.$ or $\left.N_{n}\right)$ with $N_{n}$ or $\left(P_{n}\right)$ and equate the result with predefined value.

Step-7: Increase $\mathrm{Z}=\mathrm{Z}+1$ when result is equal to $\mathrm{X}$ and Separate those subsets which are equal to $\mathrm{x}$ and make a set.

Step-8: Check all possible of combinations are done

Step-9: then check Z value if it is Zero display 'NO SUBSETS' otherwise "Display all Possible Subsets'. Then Go to Step-10

Step-10: Terminate (or END) the Program

\section{FLOW CHART FOR JEEVAN - KUSHALAIAH ALGORITHM}

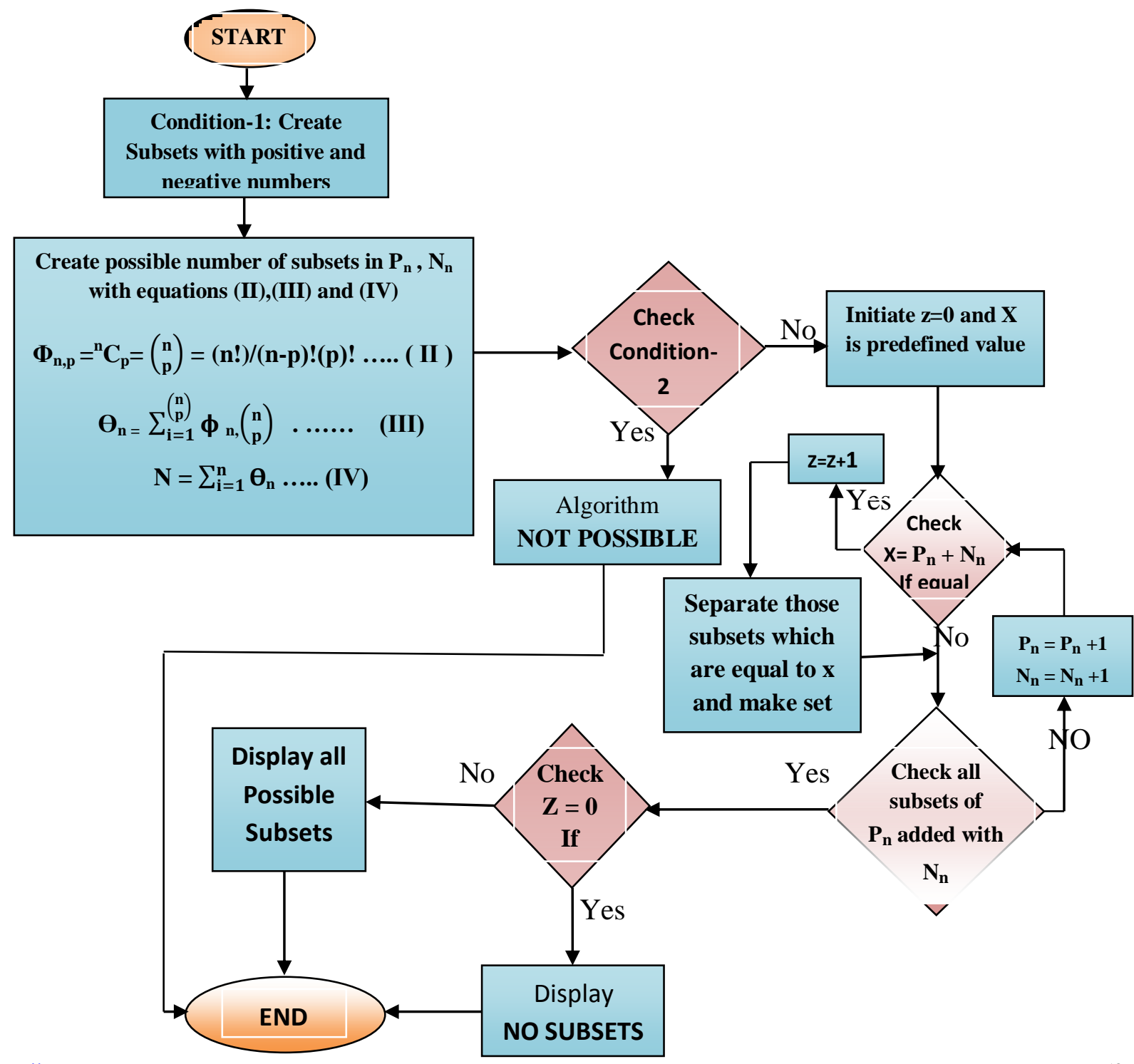


- Number of iterations of algorithm $=$ [no.of subsets of $\left.\mathbf{P}_{\mathbf{n}}\right]^{*}$ [ no.of subsets of $\left.\mathbf{N}_{\mathbf{n}}\right] \quad \ldots$ ( V)

- The area of Jeevan - Kushalaiah Algorithm table is $\mathrm{NxN}$ (Where $\mathrm{N}$ is from equation (IV))

\section{COMPUTATIONAL TIME}

The total computational time[6-7] $\mathbf{T}(\mathbf{n})=$ EXPTIME(P) is directly proportionate to $\mathrm{N}_{1} * \mathrm{~N}_{2}$ (time taken for each addition)

Where EXPTIME = exponential time[8] for calculating subset elements of $\mathrm{P}_{\mathrm{n}}$ and $\mathrm{N}_{\mathrm{n}}$

and $\mathrm{P}=$ polynomial time[9] for calculating arithmetic operation (i.e., addition) between two elements between $\mathrm{P}_{\mathrm{n}}$ and $\mathrm{N}_{\mathrm{n}}$

$\mathrm{N}_{1}=$ Number of subsets of $\mathrm{P}_{\mathrm{n}}$ and $\mathrm{N}_{2}$ is number of subsets of $\mathrm{N}_{n}$

EXPTIME $=2^{\text {poly(n1) }} \quad \ldots . . . . . . . .$. (from equation I.a)

$P=2^{O(\log n 2)} \ldots \ldots \ldots \ldots . . . .($ from equation $I . b)$

T(n) $=$ EXPTIME $(\mathbf{P})=$ $\left(2^{\text {poly(n1) }}\right)\left(2^{\mathrm{O}(\log n 2)}\right)$

The arithmetic time is directly proportional to $\mathrm{N}_{1} *$ $\mathrm{N}_{2}=\mathrm{n}_{2}$

T(n) $\alpha$ EXPTIME(n $\left(n_{2}\right) \ldots . . .(V I . b)$

\section{EXAMPLE}

Does a subset of the set $\{-2,-3,15,14,7$, $-10\}$ add up to $x=0$ ?

\section{Checking Jeevan-Kushalaiah Algorithm Conditions}

Condition-1: Create Subsets with positive and negative numbers and name as $\mathrm{P}_{\mathrm{n}}$ and $\mathrm{N}_{\mathrm{n}}$ respectively

$$
P_{n}=\{15,14,7\} \text { and } N_{n}=\{-2,-3,-10\}
$$

Condition-2: The sum all values of either set should not be less than minimum value of other set

$\operatorname{Mod}\left\{\sum \mathbf{P}_{\mathbf{n}}\right\} \operatorname{not}<\operatorname{Mod}\left\{\min \left[\mathbf{N}_{\mathbf{n}}\right]\right\} \quad$ or $\operatorname{Mod}$ $\left\{\sum \mathbf{N}_{\mathbf{n}}\right\} \operatorname{not}<\operatorname{Mod}\left\{\min \left[\mathbf{P}_{\mathbf{n}}\right]\right\}$

$\operatorname{Mod}(14+14+7)>\operatorname{Mod}(-2) \quad$ and $\operatorname{Mod}(-2-3-10)$ $<\operatorname{Mod}(7)$, Condition -2 is satisfied
Condition-3: Eliminate or remove or delete $\Theta_{0}$ value from $\mathrm{P}_{\mathrm{n}}$ and $\mathrm{N}_{\mathrm{n}}$

$P_{n}=\left\{\Theta_{1}, \Theta_{2}, \Theta_{3}\right\}$ and $N_{n}=\left\{\Theta_{1}, \Theta_{2}, \Theta_{3}\right\}$ : number of subsets, $\mathrm{N}=7$ from equations (I),(II) and (III)

$\mathrm{P}_{\mathrm{n}}$

$\{\quad[15],[14],[7],[(15+14=29),(15+7=22),(14+7=21)],[15$

$+14+7=36]\}=\{[15],[14],[7],[(29),(22),(21)][36]\}$

$\mathrm{N}_{\mathrm{n}}=\{[-2],[-3],[-10],[(-2-3=-5),(-2-10=-12),(-3-10=-$

$13)],[-2-3-10=-15]\}=\{[-2],[-3],[-10],[(-5),(-$

12), (13)],[-15]\}

$\mathbf{P}_{\mathbf{n}}: \Theta_{1}=[(15),(14),(7)], \Theta_{2}=[(29),(22),(21)], \Theta_{3}=$ [36]; $\mathbf{N}_{\mathbf{n}}: \Theta_{1}=[(-2),(-3),(-10)], \Theta_{2}=[(-5),(-12),(-13)]$, $\Theta_{3}=[-15]$

By the algorithm flow chart equation - (VI):

Number of iteration $=\left[\text { no.of subsets of } \mathbf{P}_{\mathbf{n}}\right]^{*}[$ no.of subsets of $\left.\mathbf{N}_{\mathbf{n}}\right]=[7]^{*}[7]=49$

\section{CONCLUSION}

The manuscript explained and proposed about Subsets of sets which are equal to predefined value, $\mathrm{X}$ by adding. The elements of the Set are either Constants like Trigonometry, Logarithmic, Exponential Functions or Integers like $\mathrm{K} 1, \mathrm{~K} 2 \ldots$.. Any type of arithmetic operations like addition, subtraction, multiplication and division is performed on the sets. Many computer scientists believe that $\mathrm{P} \neq \mathrm{NP}$ and Author too believe. The main reasons for failing of Jeevan - Kushalaiah algorithm is conditions on sets and Computational Program for Set. Even though all of them are satisfied there may be a chance of getting solution with $\mathrm{Z}=0$ (zero) its mean the solution is cannot be determined by Computer program but can be calculated the paper work and major drawback is there is no Algorithm program in the world to perform operation on sets. The Example shows only for 6-element set as the elements of subset increases the complexity of the problem increases Exp (poly)..ly of computational time, T(n).

Table.1 : Jeevan - Kushalaiah Algorithm table to get Predefined value, $\mathrm{X}=\mathrm{Pn}+\mathrm{Nn}$ 


\begin{tabular}{|c|c|c|c|c|c|c|}
\hline $\begin{array}{c}X=P_{n}+N_{n}= \\
0\end{array}$ & $X=P_{n}+N_{n}=0$ & $\begin{array}{c}X=P_{n}+N_{n}= \\
0\end{array}$ & $X=P_{n}+N_{n}=0$ & $X=P_{n}+N_{n}=0$ & $X=P_{n}+N_{n}=0$ & $\begin{array}{c}X=P_{n}+N_{n}= \\
0\end{array}$ \\
\hline $\begin{array}{l}15-2=13 \\
15-3=12 \\
15-10=5 \\
15-5=10 \\
15-12=3 \\
15-13=2 \\
15-15=0 *\end{array}$ & $\begin{array}{l}14-2=12 \\
14-3=11 \\
14-10=4 \\
14-5=19 \\
14-12=1 \\
14-13=1 \\
14-15=-1\end{array}$ & $\begin{array}{l}7-2=5 \\
7-3=4 \\
7-10=-3 \\
7-5=2 \\
7-12=-5 \\
7-13=-6 \\
7-15=-8\end{array}$ & $\begin{array}{l}29-2=27 \\
29-3=26 \\
29-10=4 \\
29-5=24 \\
29-12=17 \\
29-13=16 \\
29-15=14\end{array}$ & $\begin{array}{l}22-2=20 \\
22-3=19 \\
22-10=12 \\
22-5=17 \\
22-12=10 \\
22-13=9 \\
22-15=7\end{array}$ & $\begin{array}{l}21-2=19 \\
21-3=18 \\
21-10=11 \\
21-5=16 \\
21-12=9 \\
21-13=8 \\
21-15=6\end{array}$ & $\begin{array}{l}36-2=34 \\
36-3=33 \\
36-10=26 \\
36-5=29 \\
36-12=24 \\
36-13=23 \\
36-15=21\end{array}$ \\
\hline
\end{tabular}

Subsets which are equal to $\mathbf{X}=\mathbf{P}_{\mathbf{n}}+\mathbf{N}_{\mathbf{n}}=$ predefined value (i.e., $\mathbf{0}$ )

ANSWER is $\{15,-2,-3,-10\}$

\section{REFERENCES}

[1]. Cook, Stephen, "The $\mathbf{P}$ versus NP Problem". Clay Mathematics Institute. April 2000, Retrieved 18 October 2006

[2]. Cook, Stephen "The complexity of theorem proving procedures". Proceedings of the Third Annual ACM Symposium on Theory of Computing. 1971, pp. 151-158.

[3]. Ben-David, Shai; Halevi, Shai (1992). On the independence of $\mathrm{P}$ versus NP. Technical Report 714. Technion

[4]. Elvira Mayordomo. "P versus NP" Monografías de la Real Academia de Ciencias de Zaragoza 26: 5768 (2004)

[5]. Neelam Jeevan Kumar, Neelam Kushalaiah, "JEEVAN-KUSHALAIAH METHOD TO FIND THE COEFFICIENTS OF CHARACTERISTIC EQUATION OF A MATRIX AND INTRODUCTION OF SUMMETOR", IJSER, pp 1553-1562, 4(8), ISSN 2229-5518, Aug-2013

[6]. Lance Fortnow, Steve Homer, "The Computational Complexity Column", NEC Laboratories America, Princeton, NJ 08540, USA.

[7]. Christos Papadimitriou "Computational Complexity”. Addison-Wesley. ISBN 0-201-530821. 1991, page 491.

[8]. J. M. Robson, "N by $\mathrm{N}$ checkers is Exptime complete". SIAM Journal on Computing, 1984, 13 (2): 252-267

[9]. Terr, David. "Polynomial Time." From MathWorld-A Wolfram Web Resource, created by Eric W. Weisstein.

[10].Impagliazzo, R.; Paturi, R.; Zane, F. "Which problems have strongly exponential complexity?", Journal of Computer and System Sciences, 2001, 63 (4): 512-530

Wikipedia Links:

[1]. http://en.wikipedia.org/wiki/Millennium_Prize_Pro blems

[2]. http://en.wikipedia.org/wiki/P_versus_NP_problem
[3]. http://en.wikipedia.org/wiki/EXPTIME

[4]. http://en.wikipedia.org/wiki/Polynomial_time\#Poly nomial_time

Books:

[1]. Christos Papadimitriou, "Computational Complexity”. Addison-Wesley. ISBN 0-201-530821. 1991, page 491

[2]. Schrijver, Alexander, "Preliminaries on algorithms and Complexity". Combinatorial Optimization: Polyhedra and Efficiency 1, 2003, Springer. ISBN 3-540-44389-4.

[3]. Carlson, James; Jaffe, Arthur; Wiles, Andrew, eds. (2006). The Millennium Prize Problems. Providence, RI: American Mathematical Society and Clay Mathematics Institute. ISBN978-0-8218$3679-8$ 